\title{
CCD photometry and new models of 5 minor planets
}

\author{
L.L. Kiss ${ }^{1,3}$, Gy. Szabó ${ }^{1,3}$, and K. Sárneczky ${ }^{2,3}$ \\ 1 Department of Experimental Physics \& Astronomical Observatory, JATE University, H-6720 Szeged, Dóm tér 9., Hungary \\ 2 Department of Physical Geography, ELTE University, H-1088 Budapest, Ludovika tér 2., Hungary \\ ${ }^{3}$ Guest Observer at Konkoly Observatory, Hungary
}

Received June 28; accepted September 6, 1999

\begin{abstract}
We present new $R$ filtered CCD observations of 5 faint and moderately faint asteroids carried out between October, 1998 and January, 1999. The achieved accuracy is between $0.01-0.03 \mathrm{mag}$, depending mainly on the target brightness. The obtained sinodic periods and amplitudes: 683 Lanzia $-4^{\mathrm{h}} .6 \pm 0.2,0.13 \mathrm{mag} ; 725$ Amanda $>3.0, \geq 0.40$ mag; 852 Wladilena $-4.62 \pm 0.01,0.32 \mathrm{mag}$ (December, 1998) and 0.27 mag (January, 1999); 1627 Ivar $-4.80 \pm 0.01$, $0.77 \mathrm{mag}$ (December, 1998) and $0.92 \mathrm{mag}$ (January, 1999). The Near Earth Object 1998 PG unambiguously showed doubly-periodic lightcurve, suggesting the possibility of a relatively fast precession $\left(P_{1}=1.3\right.$, $P_{2}=5.3$.

Collecting all data from the literature, we determined new models for 3 minor planets. The resulting spin vectors and triaxial ellipsoids have been calculated by an amplitude-method. Sidereal periods and senses of rotation were calculated for two asteroids (683 and 1627) by a modified epoch-method. The results are: $683-\lambda_{\mathrm{p}}=$ $15 / 195 \pm 25^{\circ}, \beta_{\mathrm{p}}=52 \pm 15^{\circ}, a / b=1.15 \pm 0.05, b / c=$ $1.05 \pm 0.05, P_{\text {sid }}=0.1964156 \pm 0.0000001$, retrograde; $852-\lambda_{\mathrm{p}}=30 / 210 \pm 20^{\circ}, \beta_{\mathrm{p}}=30 \pm 10^{\circ}, a / b=$ $2.3 \pm 0.3, b / c=1.2 \pm 0.2 ; 1627-\lambda_{\mathrm{p}}=145 / 325 \pm 8^{\circ}$, $\beta_{\mathrm{p}}=34 \pm 6^{\circ}, a / b=2.0 \pm 0.1, b / c=1.09 \pm 0.05$, $P_{\text {sid }}=0.1999154 \pm 0.0000003$, retrograde. The obtained shape of 1627 is in good agreement with radar images by Ostro et al. (1990).
\end{abstract}

Key words: solar system: general minor planets

\section{Introduction}

Ground-based modelling of the shape and the rotation of the minor planets requires high precision and longterm photometric observations. With the advent of the CCD era it has become possible to study much fainter

Send offprint requests to: l.kiss@physx.u-szeged.hu minor planets than previously. The photometric methods of modelling are based on multi-opposition lightcurves of full phase coverage obtained at very different longitudes (De Angelis 1993; Detal et al. 1994; Szabó et al. 1999). Another important aspect is to detect possible collisional effects, e.g. multiperiodic lightcurves due to binarity or precession, as they may yield insights into the recent solar system evolution.

We started a long-term observational project addressed to photometric monitoring of selected minor planets. The observing programme consists of asteroids with available multi-opposition lightcurves enabling application of different photometric methods in order to model their shape and rotation. First results of this project have already been published in Sárneczky et al. (1999) and Szabó et al. (1999). The main aim of this paper is to present new CCD observations carried out between October, 1998 and January, 1999 and models for 3 minor planets. Observations, their limitations and applied methods are discussed in Sect. 2, while Sect. 3 deals with the detailed observational results.

\section{Observations and modelling methods}

We carried out $R_{\mathrm{C}}$ filtered CCD observations at Piszkéstető Station of Konkoly Observatory on ten nights from October, 1998 to January, 1999. The data were obtained using the 60/90/180 cm Schmidt-telescope equipped with a Photometrics AT200 CCD camera $(1536 \times 1024 \mathrm{KAF} 1600 \mathrm{MCII}$ coated CCD chip). The projected sky area is $29^{\prime} \times 18^{\prime}$ which corresponds to an angular resolution of 1 .' $1 /$ pixel.

The exposure times were limited by two factors: firstly, the asteroids were not allowed to move more than the FWHM of the stellar profiles (varying from night to night) and secondly, the signal-to-noise $(\mathrm{SN})$ ratio had to be at least 10. This latter parameter was estimated by comparing the peak pixel values with the sky background during 
Table 1. The journal of observations. $(r-$ geocentric distance; $\Delta$ - heliocentric distance; $\lambda$ - ecliptic longitude; $\beta$ - ecliptic latitude; $\alpha$ - solar phase angle; aspect data are referred to 2000.0)

\begin{tabular}{|c|c|c|c|c|c|c|c|}
\hline Date & $\mathrm{RA}$ & Decl. & $r(\mathrm{AU})$ & $\Delta(\mathrm{AU})$ & $\lambda$ & $\beta$ & $\alpha$ \\
\hline \multicolumn{8}{|l|}{683 Lanzia } \\
\hline 199812 14/15 & 0012.78 & +1958.9 & 3.25 & 2.82 & 20 & 18 & 17 \\
\hline 199812 16/17 & 0013.78 & +1949.5 & 3.25 & 2.84 & 20 & 18 & 17 \\
\hline \multicolumn{8}{|l|}{725 Amanda } \\
\hline 199901 26/27 & 0631.61 & +2713.5 & 2.34 & 1.44 & 110 & 24 & 12 \\
\hline \multicolumn{8}{|l|}{852 Wladilena } \\
\hline 199812 12/13 & 1140.37 & +2728.2 & 2.98 & 2.71 & 163 & 23 & 19 \\
\hline 199812 14/15 & 1141.67 & +2731.9 & 2.98 & 2.68 & 163 & 23 & 19 \\
\hline 199812 16/17 & 1142.89 & +2736.2 & 2.98 & 2.65 & 163 & 23 & 19 \\
\hline $19990124 / 25$ & 1147.52 & +3052.9 & 2.95 & 2.18 & 170 & 19 & 14 \\
\hline \multicolumn{8}{|l|}{1627 Ivar } \\
\hline 199812 14/15 & 0503.41 & +1030.4 & 2.22 & 1.26 & 76 & -12 & 6 \\
\hline 199812 15/16 & 0502.00 & +1032.6 & 2.23 & 1.26 & 76 & -12 & 6 \\
\hline 199812 16/17 & 0500.62 & +1034.9 & 2.23 & 1.27 & 76 & -12 & 7 \\
\hline $19990122 / 23$ & 0430.95 & +1309.2 & 2.35 & 1.65 & 85 & -13 & 20 \\
\hline \multicolumn{8}{|l|}{1998 PG } \\
\hline 199810 23/24 & 2347.69 & +09 15.0 & 1.23 & 0.26 & 2 & 9 & 25 \\
\hline $19981026 / 27$ & 2355.11 & +0826.9 & 1.23 & 0.27 & 2 & 7 & 25 \\
\hline $19981027 / 28$ & 2357.63 & +0811.6 & 1.23 & 0.27 & 2 & 7 & 26 \\
\hline
\end{tabular}

the observations. The journal of observations is summarized in Table 1.

The image reduction was done with standard IRAF routines. The relatively high electronic noises and low angular resolution did not permit the use of psf-photometry and that is why a simple aperture photometry was performed with the IRAF task noao.digiphot.apphot.qphot. Unfortunately other filters were not available during the observing run and consequently we could obtain only instrumental differential $R$ magnitudes in respect to closely separated comparison stars. The precision was estimated with the rms scatter of the comp.-check magnitudes (tipically $0.01-0.03 \mathrm{mag})$.

We have also investigated the possible colour effects in neglecting standard photometric transformations. We made an $R$ filtered 60-seconds CCD image of open cluster M67 on December 14, 1998. This cluster contains a widely used sequence of photometric standard stars (Schild 1983). We determined the instrumental magnitude differences in respect to star No. 81 in Schild (1983), which various colour indices are close to zero $((B-V)=-0.098$, $\left.(V-R)_{\mathrm{C}}=-0.047 \mathrm{mag}\right)$. The studied standards were stars Nos. 106, 108, 117, 124, 127, 128, 129, 130, 134 and 135, following Schild's notation. We plotted the resulting differences $\left(\Delta R_{\text {ins }}-\Delta R_{\text {std }}\right)$ vs. $(B-V)$ and $(V-R)_{\mathrm{C}}$ in Fig. 1. For a wide colour range they do not differ more than $0.1 \mathrm{mag}$, while the colour dependence is quite weak. Therefore, the obtained instrumental $R$-amplitudes of minor planet lightcurves are very close to the standard ones, allowing reliable comparison with other measurements.

The presented magnitudes throughout the paper are based on magnitudes of the comparison stars taken from the Guide Star Catalogue (GSC) (Table 2). Therefore,
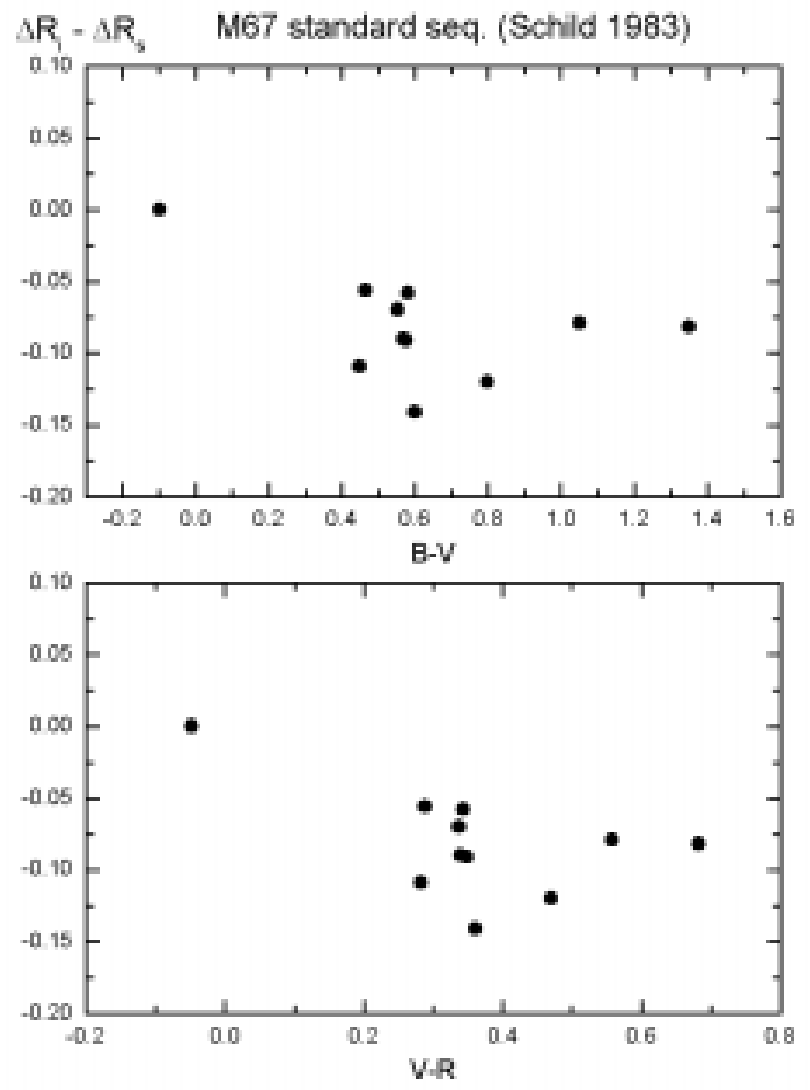

Fig. 1. The colour dependence of instrumental minus standard magnitude differences for selected photometric standard stars in M67 
Table 2. The comparison stars. The typical uncertainty in the magnitude values is as large as $\pm 0.2-0.3 \mathrm{mag}$

\begin{tabular}{lll}
\hline Date & Comp. & $m$ (GSC) \\
\hline 683 Lanzia & & \\
19981214 & GSC 1182 337 & 15.3 \\
19981216 & GSC 1182 85 & 14.4 \\
725 Amanda & & \\
1999 01 26 & GSC 1887 1325 & 12.3 \\
852 Wladilena & & \\
19981212 & GSC 1984 2286 & 12.7 \\
19981214 & GSC 1984 2516 & 12.0 \\
19981216 & GSC 1984 2496 & 13.8 \\
19990124 & GSC 2524 1778 & 12.6 \\
1627 Ivar & & \\
19981212 & GSC 702 759 & 12.6 \\
19981214 & GSC 689 1331 & 12.8 \\
19981216 & GSC 689 2101 & 12.6 \\
19990122 & GSC 681 519 & 13.7 \\
1998 PG & & \\
19981023 & GSC 1170 1119 & 14.2 \\
19981024 & GSC 1171 632 & 14.3 \\
19981026 & GSC 1171 1424 & 14.5 \\
\hline
\end{tabular}

their absolute values are fairly uncertain (at level of $\pm 0.2-0.3 \mathrm{mag}$ ). Fortunately it does not affect the other photometric parameters needed in the minor planet studies, such as the amplitude, time of extrema, or photometric period. The final step in the data reduction was the correction for the light time ${ }^{1}$. Composite diagrams were calculated using APC11 by Jokiel (1990) and are also light time corrected. Times of zero phase are included in the individual remarks.

Two methods were applied for modelling. The first is the well-known amplitude-method described, e.g., by Magnusson (1989) and Michałowski (1993). For this the amplitude information is used to determine the spin vector and the shape. An important point is that the observed $A(\alpha)$ amplitudes at solar phase $\alpha$ should be reduced to zero phase $\left(A\left(0^{\circ}\right)\right)$, if possible, by a simple linear transformation in form of $A(\alpha)=A\left(0^{\circ}\right)(1+m \alpha) . m$ is a parameter, which has to be determined individually and that can be difficult, or even impossible if there are insufficient observations (Zappala et al. 1990).

The other possibility is to examine the times of light extrema ("epoch-methods", "E-methods"). In this paper a modified version was used, which gives the sense of the rotation unambiguously. The pole coordinates can be also estimated independently. Further details can be found in Szabó et al. (1999) and Szabó et al. (in preparation), here we give only a brief description.

The initial idea is that the prograde and retrograde rotation can be distinguished by following the virtual shifts of moments of light extrema (e.g. times of minima). From

\footnotetext{
${ }^{1}$ Individual data are available upon request from the second author (szgy@neptun.physx.u-szeged.hu).
}

a geocentric point of view, a full revolution around the Earth causes one extra rotational cycle to be added (retrograde rotation) or subtracted (prograde rotation) to the observed number of rotational cycles during that period. The virtual shifts increase or decrease monotonically and their cumulative change is exactly one period over one revolution. Therefore, plotting the observed minus calculated $(\mathrm{O}-\mathrm{C})$ times of minima versus the geocentric longitude, we get a monotone function ascending or descending by the value of the period. The definition of the observed $\mathrm{O}-\mathrm{C}$ is as follows:

$$
\mathrm{O}-\mathrm{C}=T_{\min }-\left(E_{0}+N \cdot P_{\text {sid }}\right) \equiv\left\langle\frac{\Delta T}{P_{\text {sid }}}\right\rangle P_{\text {sid }}
$$

where $T_{\min }$ means the observed time of minimum, $E_{0}$ is the epoch, while the $N$ integer number denotes the cycles (e.g. the number of rotation) of $P_{\text {sid }}$ period between the observed extremum and the epoch. $\Delta T$ means the time interval between $T_{\min }$ and $E_{0}$, and \langle\rangle denotes fractional part. The theoretical $\mathrm{O}-\mathrm{C}$ curve depends on the pole coordinates:

$\mathrm{O}-\mathrm{C}^{\prime}:=\frac{\mathrm{O}-\mathrm{C}}{P_{\text {sid }}}=\frac{1}{\pi} \arctan \frac{\tan \left(\Lambda-\lambda_{\mathrm{p}}\right)}{\sin \left(\beta_{\mathrm{p}}-B\right)}$

where $\Lambda$ and $B$ denote geocentric longitude and latitude; $\lambda_{\mathrm{p}}$ and $\beta_{\mathrm{p}}$ are the pole coordinates.

The main difference between the classical E-methods and this $\mathrm{O}-\mathrm{C}^{\prime}$ method is that time dependence is transformed into the geocentric longitude domain. Because of the system's basic symmetries, the $\mathrm{O}-\mathrm{C}^{\prime}$ diagrams are calculated for a half revolution and with the half sidereal period. The fitting procedure consists of altering $P_{\text {sid }}$ until the observed times of minima do not give a monotone $\mathrm{O}-\mathrm{C}^{\prime}$ diagram showing an increase or decrease of exactly 1. Fitting a theoretical curve (Eq. 2) to the observed points, the pole coordinates can be also estimated.

\section{Discussion}

\section{Lanzia}

This minor planet was discovered by M. Wolf in Heidelberg, on July 23, 1909. It was observed in the 1979, 1982, 1983-1984, 1987 oppositions (Carlsson \& Lagerkvist 1981; Weidenschilling et al. 1990). Carlsson \& Lagerkvist (1981) determined a rotation period of 4. 322 and an amplitude of 0.14 mag. On the other hand, Weidenschilling et al. (1990) measured a period of 4.37 with an amplitude of 0.12 mag.

Our observations in 1998 suggest a period of $4.6 \pm 0.2$ with an amplitude of $0.13 \pm 0.01$. Composite diagrams calculated with previously published periods between $4.3-4.4$ have much larger scatter. The light-time corrected composite diagram is presented in Fig. 2. The zero phase is JD 2451162.3169.

Based on earlier data (see Table 3), a new model has been determined with amplitude method. The observed 


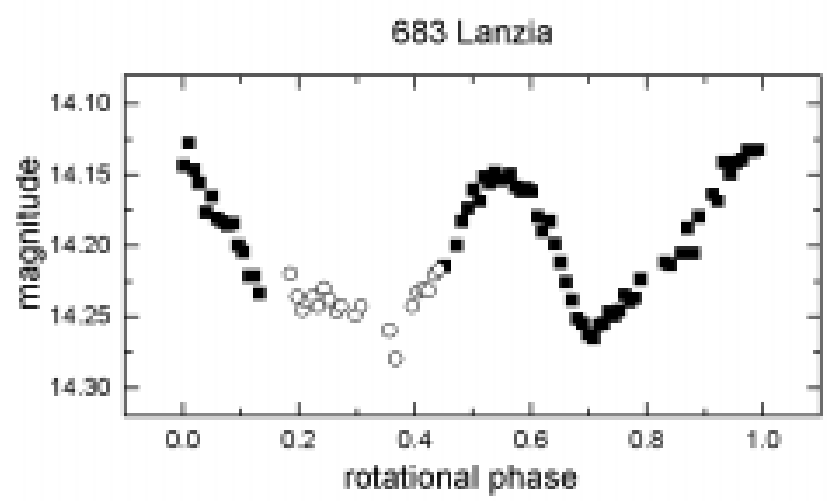

Fig. 2. The composite $R$ lightcurve of 683 (symbols: solid squares - December 14; open circles - December 16)

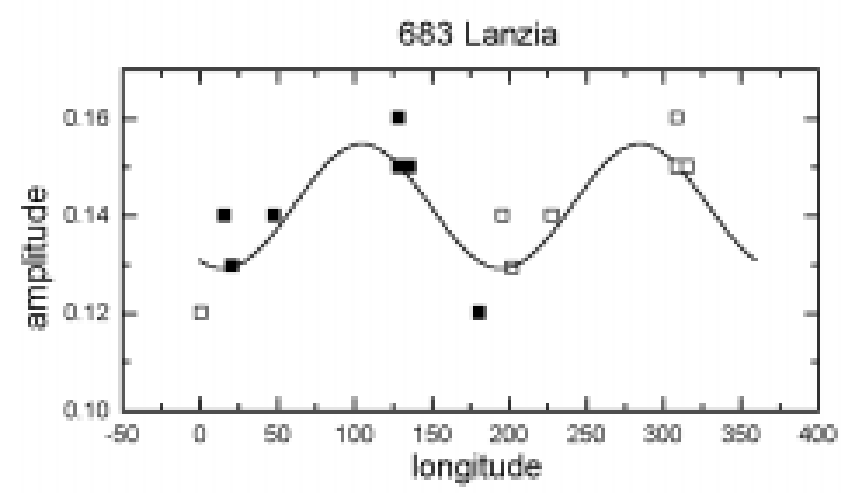

Fig. 3. The observed amplitudes vs. longitudes with the determined fit for 683 Lanzia

amplitudes vs. ecliptic longitudes with the fit are plotted in Fig. 3. The resulting triaxial ellipsoid has the following parameters: $a / b=1.15 \pm 0.07, b / c=1.05 \pm 0.05$, while the spin vector's coordinates are $\lambda_{\mathrm{p}}=15 / 195 \pm 25^{\circ}$, $\beta_{\mathrm{p}}=52 \pm 15^{\circ}$, respectively. We could not reduce the observed amplitudes to zero solar phase, since the actual value of $m$ parameter (e.g. Zappala et al. 1990) could not be estimated by the data sequence or asteroid classification. Also we have to note that a mixture of $V$ and $R$ amplitudes was used, thus the model should be considered as an approximate one. The $\mathrm{O}-\mathrm{C}^{\prime}$ model has also been determined (Fig. 4). For reducing the errors, lightcurves obtained between October, 1983 and February, 1984, were composed and one time of minimum was determined from this composite lightcurve. The resulting sidereal period is $P_{\text {sid }}=0.1964156 \pm 0.0000001$ with retrograde rotation.

\section{Amanda}

It was discovered by J. Palisa in Vienna, on October 21, 1911. To our knowledge, the only one photometry of 725 in the literature is that of Di Martino et al. (1994) carried out in 1985. They determined a sinodic period of 3.749 associated with a full variation of $0.3 \mathrm{mag}$. Our observations do not exclude that period, as they suggest a possible

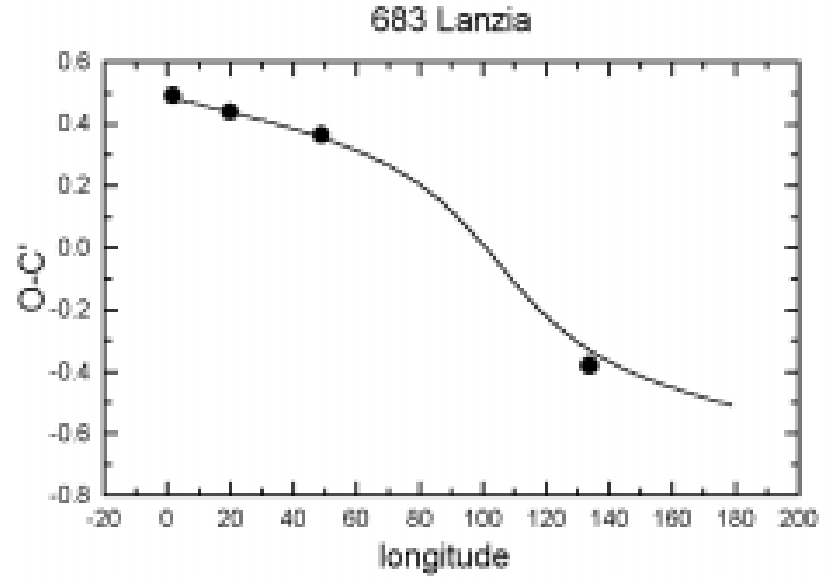

Fig. 4. The observed $\mathrm{O}-\mathrm{C}^{\prime}$ values fitted with the model for 683 Lanzia

Table 3. Published photometries of 683 Lanzia

\begin{tabular}{|c|c|c|c|c|c|c|}
\hline Date & $\lambda$ & $\bar{\beta}$ & $\alpha$ & $\bar{A}$ & $t_{\min }$ & ref. \\
\hline 19790319,20 & 182 & -27 & 9 & $0 .{ }^{\mathrm{m}} 12$ & 43963.452 & (1) \\
\hline 19821216 & 49 & 9 & 11 & 0.14 & 45319.591 & (2) \\
\hline 19831012,13 & 130 & -9 & 18 & 0.15 & 45650.871 & (2) \\
\hline 19831115 & 137 & -13 & 19 & 0.15 & 45650.871 & (2) \\
\hline 19840221 & 129 & -23 & 10 & 0.16 & 45650.871 & (2) \\
\hline 19871019 & 16 & 23 & 7 & 0.12 & 47118.538 & (2) \\
\hline 19981214,16 & 20 & 18 & 17 & 0.13 & 51162.275 & p.p. \\
\hline
\end{tabular}

References: (1) - Carlsson \& Lagerkvist (1981) (2) Weidenschilling et al. (1990).

value around 4 hours. Unfortunately the data cover only 3 hours, thus we could not draw a firm conclusion. The observations were made under fairly unfavourable conditions, which is illustrated with the comp-check curve bearing a relatively high scatter (about $\pm 0.03 \mathrm{mag}$ ). It is presented together with the observed lightcurve in Fig. 5.

\section{Wladilena}

This asteroid was discovered by S. Belyavskij in Simeis, on April 2, 1916. Its earlier photometric observations were carried out in 1977, 1982 and 1993 (Tedesco 1979; Di Martino \& Cacciatori 1984; De Angelis \& Mottola 1995). The observed light variation in 1998 had an amplitude of $0.32 \mathrm{mag}$, while the period was $4.62 \pm 0^{\mathrm{h}} .01$. This is in very good agreement with results by De Angelis \& Mottola (1995), who found a period value of 4.613 . The light time corrected composite diagram is presented in Fig. 6. The zero phase is at 2451160.5904 . The lightcurve has remarkable asymmetries - the brighter maximum is rather sharp, its hump is exactly two times shorter than the other one. There are also small amplitude, short-period humps on the longer descending branch. These phenomena can be more or less identified in the previous measurements too. That is why we carried out a second observing run on January 24, 1999. We wanted to check the reality of these 
725 Amanda

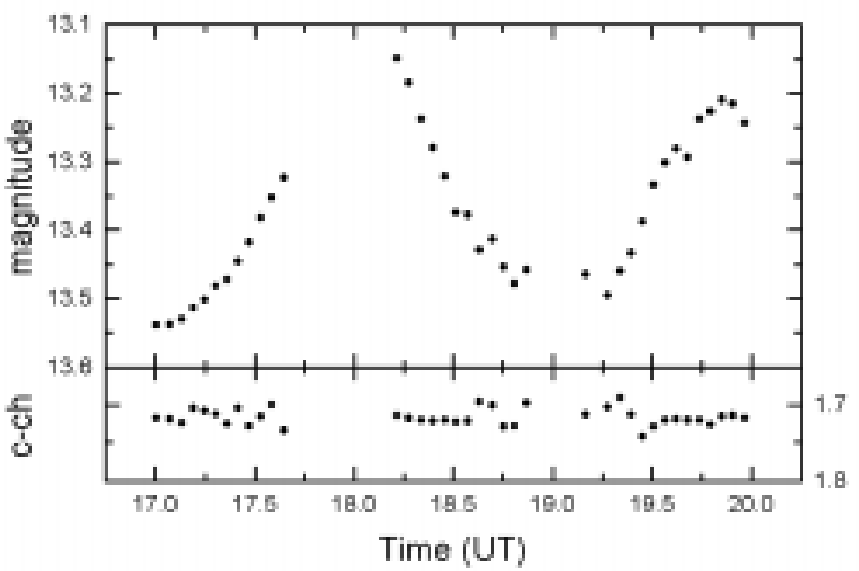

Fig. 5. The $R$ lightcurve of 725 on January 26, 1999

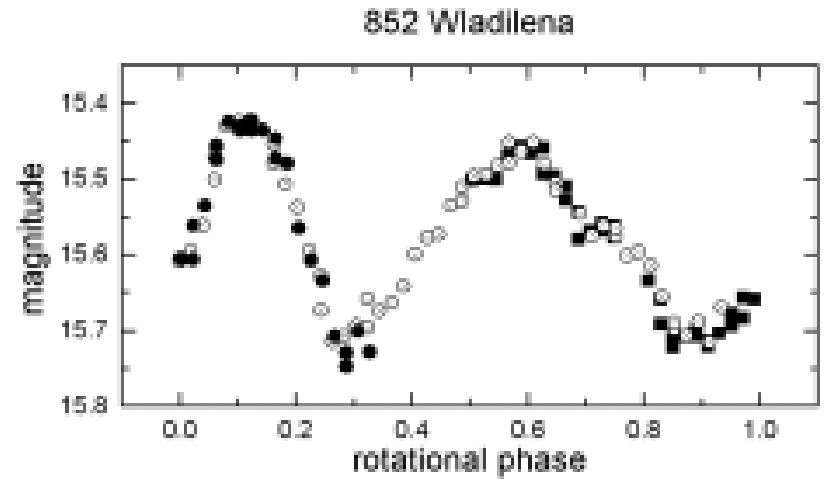

Fig. 6. The composite $R$ lightcurve of 852 (symbols: solid circles - December 12; dotted circles - December 14; solid squares December 16)

irregularities. The lightcurve revealed the same asymmetries as those of observed one month earlier (Fig. 7). This may refer to a shape with sharp asymmetries, e.g. something similar to a jagged tenpin.

We have tried to determine a new model using the earlier data summarized in Table 4. Unfortunately, the measurements have such a distribution along the longitude that reliable modelling is difficult. This is shown in Fig. 8, where the observed amplitudes vs. ecliptic longitudes are plotted with an approximate fit. The resulting parameters are as follows: $a / b=2.3 \pm 0.3, b / c=1.2 \pm 0.2$, $\lambda_{\mathrm{p}}=30 / 210 \pm 20^{\circ}, \beta_{\mathrm{p}}=30 \pm 10^{\circ}$. The pole coordinates are in considerable agreement with those of by De Angelis \& Mottola (1995), who determined two possible solutions: (1) $\lambda_{\mathrm{p}}=53 \pm 6^{\circ}, \beta_{\mathrm{p}}=24 \pm 20^{\circ}$ and $(2) \lambda_{\mathrm{p}}=235 \pm 6^{\circ}$, $\beta_{\mathrm{p}}=21 \pm 20^{\circ}$.

1627 Ivar

This Earth-approaching asteroid was discovered by E. Hertzsprung in Johannesburg, on September 25, 1929.

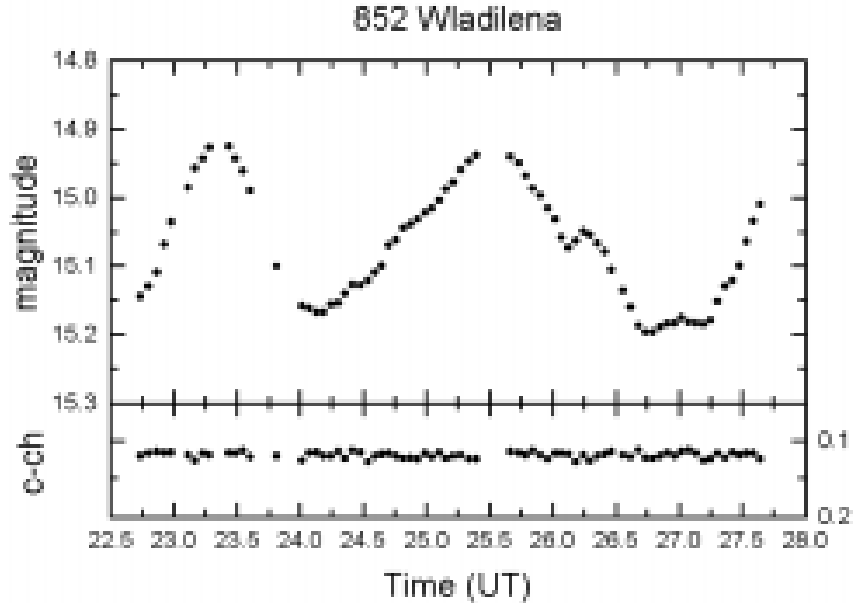

Fig. 7. The $R$ lightcurve of 852 on January 24, 1999

Table 4. Published photometries of 852 Wladilena

\begin{tabular}{|c|c|c|c|c|c|}
\hline Date & $\lambda$ & $\bar{\beta}$ & $\alpha$ & $A$ & ref. \\
\hline 19770214 & 139 & 31 & 10 & $\overline{1 . \mathrm{m}} 12$ & (1) \\
\hline 19821018 & 6 & -10 & 10 & 0.37 & (2) \\
\hline 1993118,10 & 33 & -8 & 3 & 0.23 & (3) \\
\hline $199812 \quad 12-16$ & 163 & 23 & 19 & 0.32 & p.p. \\
\hline 19990124 & 170 & 19 & 14 & 0.27 & p.p. \\
\hline
\end{tabular}

References: (1) - Tedesco (1979) (2) - Di Martino \& Cacciatori (1984) (3) De Angelis \& Mottola (1995).

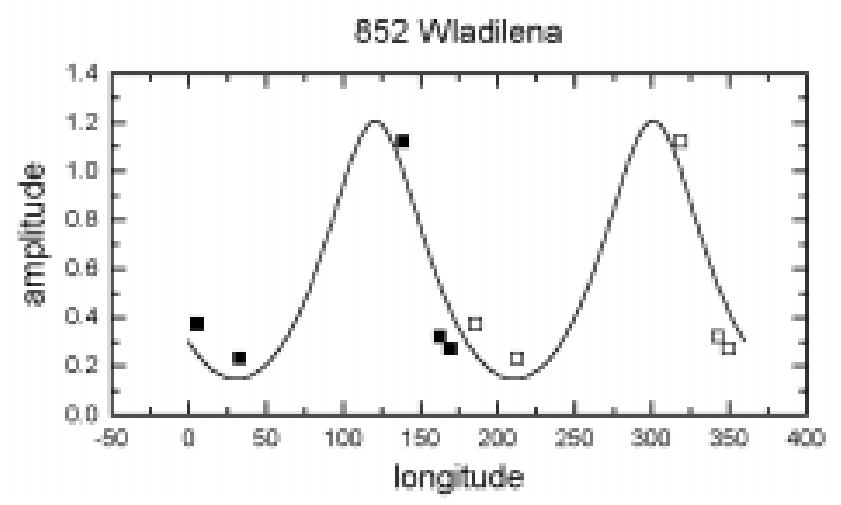

Fig. 8. The observed amplitudes vs. longitudes with the determined fit for 852 Wladilena

There are four photometric observations in the literature (Hahn et al. 1989; Velichko et al. 1990; Hoffmann \& Geyer 1990; Chernova et al. 1995) and one radar measurement by Ostro et al. (1990). The previously determined periods scatter around 4.8 , thus our resulting $4.80 \pm 0.01$ is in perfect agreement with earlier results. The amplitude changed significantly over a period of one month, as it was $0.77 \mathrm{mag}$ and $0.92 \mathrm{mag}$ in December, 1998 and January, 1999, respectively. The composite lightcurve is presented in Fig. 9, while the single lightcurve obtained in January is plotted in Fig. 10. 


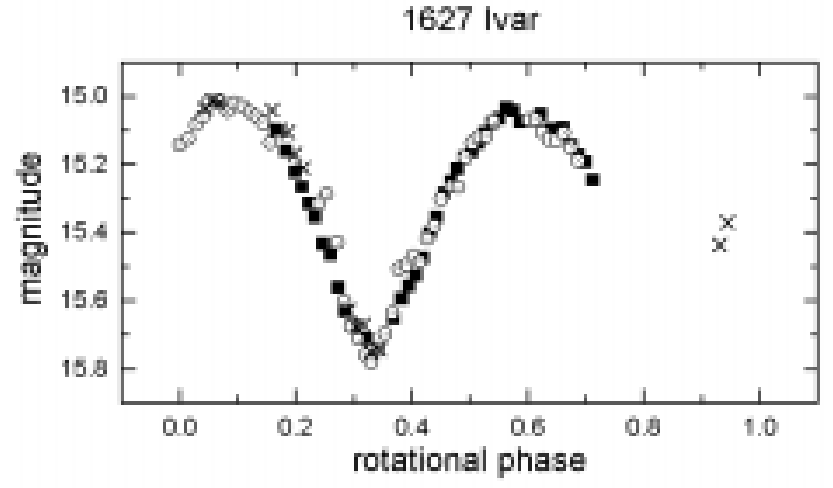

Fig. 9. The composite $R$ lightcurve of 1627 (symbols: solid squares - December 14; dotted circles - December 15; crosses - December 16)

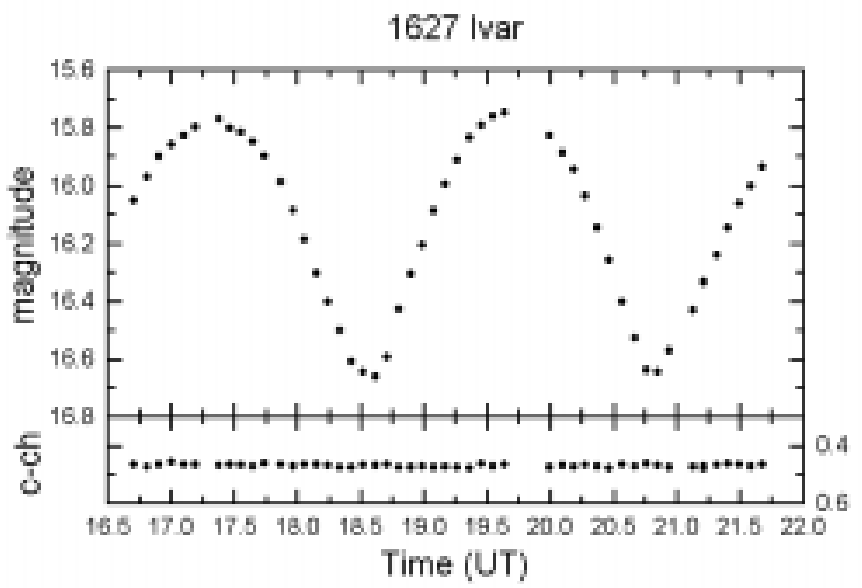

Fig. 10. The $R$ lightcurve of 1627 on January 22, 1999

A new amplitude model has been determined after collecting all available data (Table 5). The observed amplitudes were reduced to zero solar phase. First of all, the $m$ parameter was derived from our measurements. The observed amplitudes in December, 1998 and in January, 1998 were compared. As the longitudes differ by only $10^{\circ}$, and the difference between the corresponding phases is quite high $\left(13^{\circ}\right)$, the amplitude change can be mostly associated with the phase change. The result is $m=0.018$. We have also corrected other amplitudes to zero solar phase and fitted the amplitude variations along the longitude. The corresponding parameters are: $a / b=2.0 \pm 0.1$, $b / c=1.09 \pm 0.05, \lambda_{\mathrm{p}}=145 / 325 \pm 8^{\circ}, \beta_{\mathrm{p}}=34 \pm 6^{\circ}$. The reduced amplitudes with the determined fit is presented in Fig. 11. The reliability of this model was tested by a direct comparison with radar images of Ostro et al. (1990). This is shown in Fig. 12, where we used Fig. 5 taken from Ostro et al. (1990) with kind permission of the first author. The similarity is evident.

The $\mathrm{O}-\mathrm{C}^{\prime}$ method was used to determine the sidereal period and the sense of the rotation. The results are $P_{\text {sid }}=0.1999154 \pm 0.0000003$, retrograde rotation with
Table 5. Published photometries of 1627 Ivar

\begin{tabular}{lrrrlll}
\hline Date & $\lambda$ & $\beta$ & $\alpha$ & $A$ & $t_{\min }$ & ref. \\
\hline $198506 \quad 13$ & 317 & 29 & 48 & 0.35 & 46226.750 & $(1)$ \\
$198508 \quad 31$ & 15 & -21 & 32 & 0.55 & 46258.703 & $(1)$ \\
19851016 & 4 & -23 & 20 & 0.63 & 46287.184 & $(1)$ \\
$198905 \quad 01-23$ & 203 & 25 & 20 & 1.0 & 47647.402 & $(2)$ \\
$198906 \quad 15-23$ & 201 & 21 & 51 & 1.12 & 47647.402 & $(2)$ \\
$198907 \quad 14-19$ & 213 & 14 & 60 & 1.45 & 47721.565 & $(2)$ \\
$1990 \quad 0511-14$ & 204 & 25 & 24 & 1.08 & 48029.439 & $(3,4)$ \\
$1998 \quad 12 \quad 14,16$ & 76 & -12 & 79 & 0.77 & 51162.295 & p.p. \\
19990126 & 87 & -13 & 18 & 0.92 & 51201.171 & p.p. \\
\hline
\end{tabular}

References: (1) - Hahn et al. (1989) (2) - Chernova et al. (1995) (3) - Velichko et al. (1990) (4) Hoffmann \& Geyer (1990).

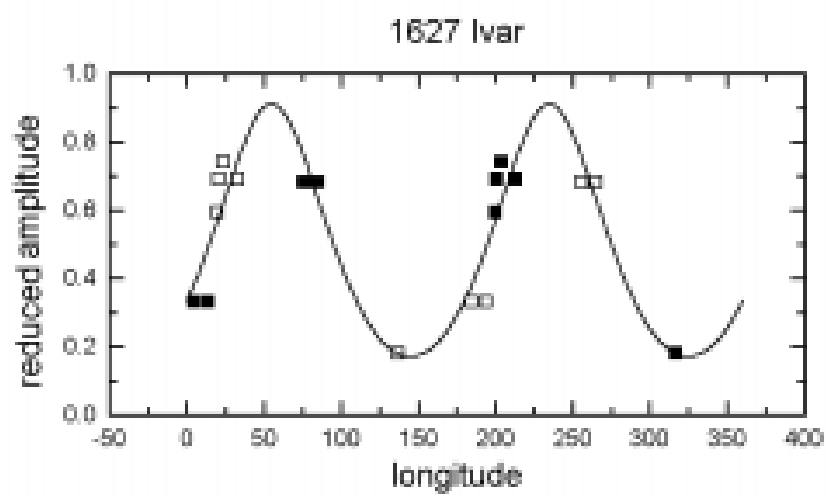

Fig. 11. The reduced amplitudes vs. longitudes with the determined fit for 1627 Ivar
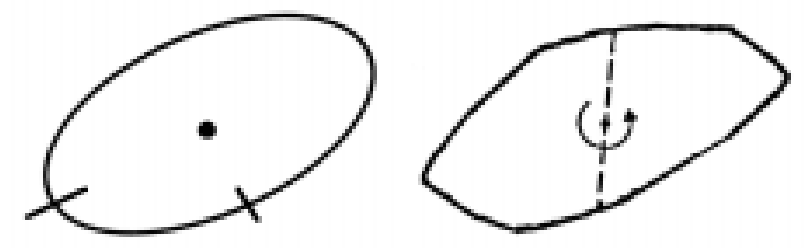

Fig. 12. A pole-on view of the photometric model (left) and radar profile (right) of 1627 Ivar. The small ticks correspond to the uncertainties of the fit

$\lambda_{\mathrm{p}}=143 \pm 8^{\circ}, \beta_{\mathrm{p}}=-37 \pm 6^{\circ}$ pole coordinates. The agreement between the poles obtained by different methods is very good. The sidereal period agrees well with results of Lupishko et al. (1986) - 0.19991, prograde -, but the senses are in contradiction. The fitted $\mathrm{O}-\mathrm{C}^{\prime}$ diagram is presented in Fig. 13.

\section{$1998 P G$}

The Near Earth Object (NEO) 1998 PG was discovered by the LONEOS project in Flagstaff, on August 3, 1998. We observed about 80 days after the discovery, in October, 1998. We found complex, strongly scattering lightcurves (two of them are shown in Figs. 14-15), which did not 
Table 6. The determined periods, amplitudes, spin vectors and shapes

\begin{tabular}{rllllllll}
\hline Asteroid & $P_{\sin }(h)$ & $P_{\text {sid }}(d)$ & $A(\mathrm{mag})$ & $\lambda_{\mathrm{p}}$ & $\beta_{\mathrm{p}}$ & $a / b$ & $b / c$ & method \\
\hline 683 & 4.6 & & 0.13 & $15 / 195 \pm 25$ & $52 \pm 15$ & $1.15 \pm 0.05$ & $1.05 \pm 0.05$ & $\mathrm{~A}$ \\
& & $0.1964156 R$ & & & & & & \\
725 & $\geq 3$ & & $\geq 0.4$ & - & - & - & $\mathrm{O}-\mathrm{C}$ \\
852 & 4.62 & & $0.32,0.27$ & $30 / 210 \pm 20$ & $30 \pm 10$ & $2.3 \pm 0.3$ & $1.2 \pm 0.2$ & $\mathrm{~A}$ \\
1627 & 4.80 & & $0.77,0.92$ & $145 / 325 \pm 8$ & $34 \pm 6$ & $2.0 \pm 0.1$ & $1.09 \pm 0.05$ & $\mathrm{~A}$ \\
& & \multirow{2}{*}{$0.1999154 R$} & & 143 & -37 & & & O-C \\
$1998 \mathrm{PG}$ & 2.6 & & 0.09 & & & & Fourier \\
- & 5.3 & & 0.08 & & & & Fourier \\
\hline
\end{tabular}

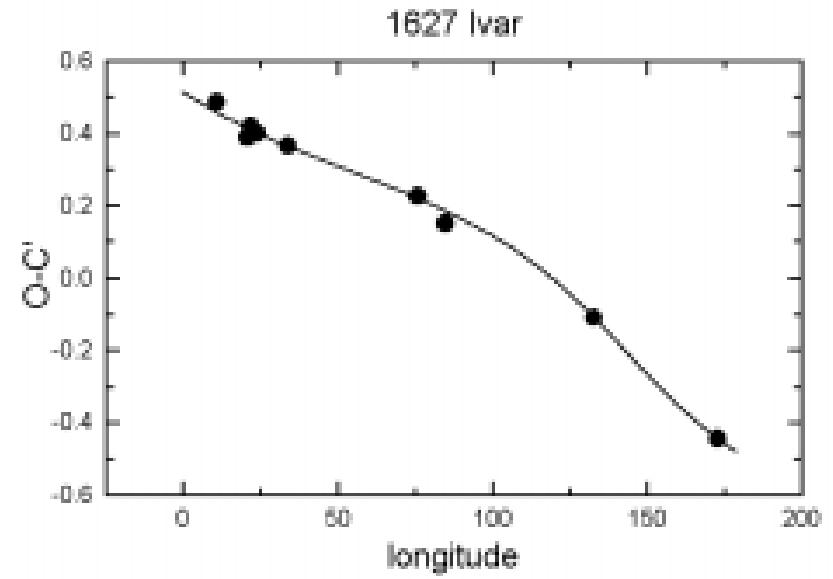

Fig. 13. The observed $\mathrm{O}-\mathrm{C}^{\prime}$ values fitted with the model for 1627 Ivar

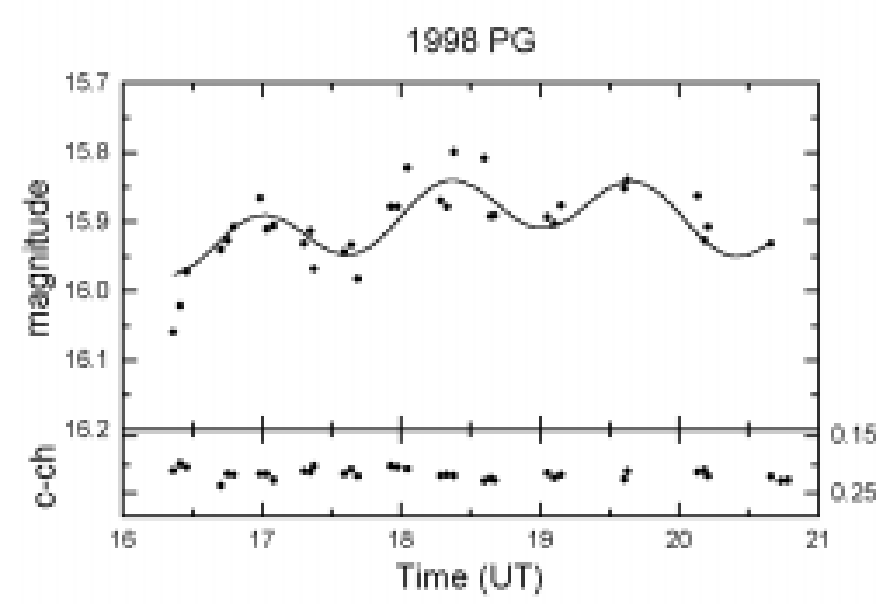

Fig. 14. The observed and fitted $R$ lightcurves of 1998 PG on October 23, 1998

show any usual regularity. Therefore, we performed a conventional frequency analysis by calculating Discrete Fourier Transform (DFT) of the whole dataset (Fig. 16). Data obtained on October 27 are too noisy, thus we excluded them from the period determination.

The determined periods are 1.3 and 5.3 , although these values have large uncertainties (about $10-15 \%$ ).

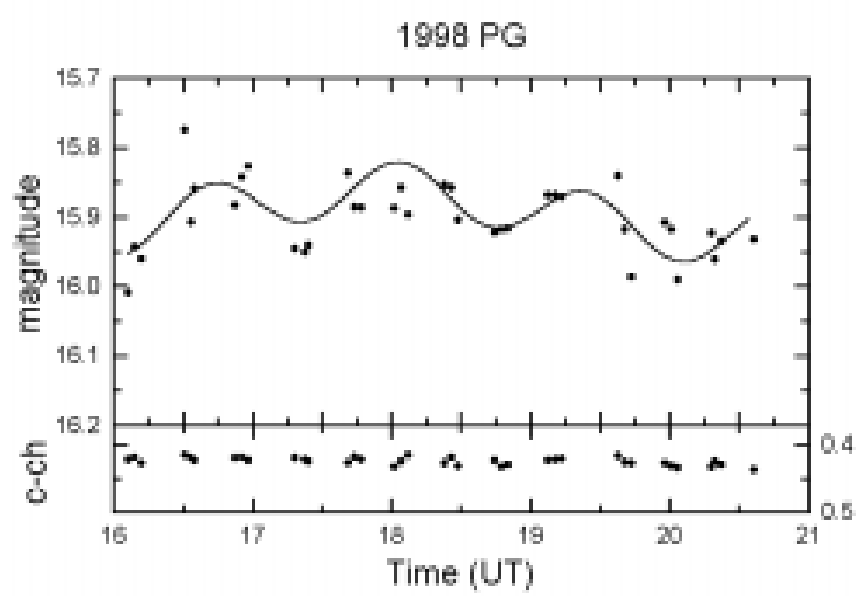

Fig. 15. The same as in Fig. 14 on October 26, 1998

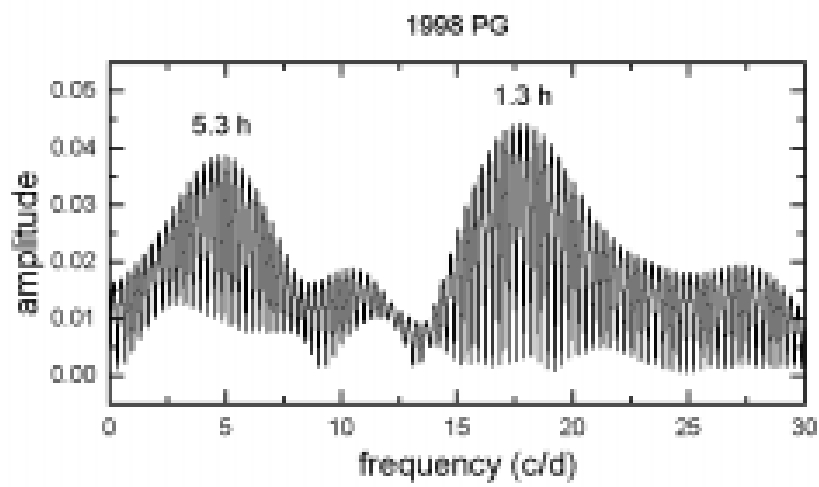

Fig. 16. Fourier spectrum of 1998 PG

Assuming that the shorter period is due to rotation, we get a rotational period of 2.6 . We note that our period values do not contradict those obtained by $\mathrm{P}$. Pravec and his collaborators, who found $P_{\text {rot }}=2.517$ and $P_{2} \approx 7$ h.0 (Pravec 1998, personal communication). The reason for doubly periodic lighcurve can be precession and/or binarity. The observed rate of multiperiodic lightcurves among NEOs is quite high (see, e.g., Pravec 1999), but the underlying physical processes can only be identified with more detailed observations than we have on 1998 PG. Therefore, we conclude that we may have found evidence for 
precession in $1998 \mathrm{PG}$, but other explanations cannot be excluded.

We summarize the resulting sinodic periods, amplitudes and models in Table 6.

Acknowledgements. This research was supported by the Szeged Observatory Foundation and OTKA Grant No. T022259. The warm hospitality of the staff of Konkoly Observatory and their provision of telescope time is gratefully acknowledged. The authors also acknowledge suggestions and careful reading of the manuscript by $\mathrm{K}$. West. The NASA ADS Abstract Service was used to access references.

\section{References}

Carlsson M., Lagerkvist C.I., 1981, A\&AS 45, 1

Chernova G.P., Kiselev N.N., Krugley Yu.N., et al., 1995, AJ 110,1875

De Angelis G., 1993, P\&SS 41, 285

De Angelis G., Mottola S., 1995, P\&SS 43, 1013

Detal A., Hainaut O., Pospieszalska-Surdej A., et al., 1994, A\&A 281, 269

Di Martini M., Cacciatori S., 1984, Icarus 60, 75

Di Martino M., Dotto E., Barucci M.A., et al., 1994, Icarus 109, 210
Hahn G., Magnusson P., Harris A.W., et al., 1989, Icarus 78, 363

Hoffmann M., Geyer E.H., 1990, AcA 40, 389

Jokiel R., 1990, Astronomical Observatory of Adam Mickiewicz University, Poznan

Lupishko D.F., Velichko F.P., Shevchenko V.G., 1986, KFNT, $2,5,39$

Magnusson P., 1989, in: Asteroids II. University of Arizona Press, p. 1180

Michałowski T., 1993, Icarus 106, 563

Ostro S.J., Campbell D.B., Hine A.A., et al., 1990, AJ 99, 2012

Pravec P., 1999, http://sunkl.asu.cas.cz/ ppravec/

Sárneczky K., Szabó Gy., Kiss L.L., 1999, A\&AS 137, 363

Szabó Gy., Sárneczky K., Kiss L.L., 1999, Contrib. Skalnate Pleso Obs. 28 (in press)

Schild R.E., 1983, PASP 95, 1021

Tedesco E.F., 1979, Ph.D. Dissertation. New Mexico State University

Velichko F.P., Krugly Y.N., Lupishko D.F., Mohamed R.A., 1990, Astron. Tsirk. 1546, 39

Weidenschilling S.J., Chapman C.R., Davis D.R., et al., 1990, Icarus 86,402

Zappala V., Cellino A., Barucci A.M., et al., 1990, A\&A 231, 548 